\title{
Accuracy of Transillumination in the Recognition of Pneumothorax and Pneumomediastinum in the Neonate
}

\author{
Michael L. Wyman, M.D., Lawrence R. Kuhns, M.D.
}

RANSILLUMINATION of the neonatal chest has been found to be a valuable diagnostic aid in several hundred neonates with acute respiratory distress, and has helped to diagnose 126 pneumothoraxes (PT) and pneumomediastina (PM) as later demonstrated radiographically. We here summarize our experiences with this technique (Table 1), and describe those factors we have found important, particularly with edematous infants.

\section{Methods}

The chests of all patients with acute respiratory symptoms in the Holden Newborn Intensive Care Unit of our hospital were transilluminated as a part of the physical examination. This was done by the attending nurses and physicians, applying the method of Kuhns et al: ${ }^{1}$ Radiographs taken subsequently were reviewed by the Department of Pediatric Radiology for the presence or absence of pneumothorax and pneumomediastinum. When present, these were graded as small, moderate or severe, primarily on the basis of radiographic size and the degree of accompanying mediastinal shift or lung collapse.

\section{Results}

Fifty neonates with radiologically demonstrable pneumomediastinum have been evalu-

\footnotetext{
From the Departments of Pediatrics and Radiology University of Michigan Medical Center, Ann Arbor, Michigan 48109 .

Correspondence to: Michael L. Wyman, M.D., 2 B 425 , University of Utah Medical Center, Salt Lake City, UT 84132
}

ated. Of these, only three were not detected by transillumination, establishing our accuracy in the diagnosis of radiographically proven pneumomediastinum as 94 per cent. These 3 cases were all small PMs that can easily be missed by transillumination, especially in large-term and post-mature neonates. Several symptomatic infants had large PMs that transilluminated not only in the substernal area, but also in a subpulmonic location, indicating extension of PM into a potential space between the parietal pleura and the diaphragm, Verification of the continuity of such collections has been provided by transillumination during single site drainage of the entire collection. Our false positive rate for either pneumomediastinum or pneumothorax ( 76 cases) is approximately 5 per cent. ${ }^{1}$

Pneumothorax (PT) is the most important condition discoverable by transillumination of the neonatal chest, and particularly when used with neonates of less than $4 \mathrm{~kg}$. We have found by post mortem injection that $10 \mathrm{ml}$ of air in the pleural space of a $1,300-\mathrm{gm}$ newborn can be plainly seen by transillumination.

\section{Discussion}

Gross chest wall edema was present in 2 of the 3 cases in which a pneumothorax was missed by light. The edema caused diffraction of the light in the chest wall of these two infants, interfering with transillumination of the intrathoracic structures. Edema enlarges the corona of light seen in the tissues, and the edge of the corona becomes indistinct. This corona is equally enlarged on both 
TABLE I Accuracy of Prior Transillimination (TI) in 126 Radiographically Proven Gases of Whobons with Pno momediastinum $(P M)$ or Pnomothorax $(P T)$

\begin{tabular}{|c|c|c|c|}
\hline Size & $\begin{array}{l}\text { Total } \\
\text { Proven } \\
\text { Cases }\end{array}$ & $\begin{array}{c}\text { T1 } \\
\text { Positive }\end{array}$ & $\begin{array}{c}\text { TI } \\
\text { Negative }\end{array}$ \\
\hline Minimal PM & 21 & 18 & 3 \\
\hline Moderate PM & 18 & 18 & 0 \\
\hline Severe PM & 11 & 11 & 0 \\
\hline All PM & 50 & 47 & 3 \\
\hline Minimal $\mathrm{PT}$ & 9 & 9 & 0 \\
\hline Moderate PT & 32 & 30 & 2 \\
\hline Severe PT & 35 & 34 & 1 \\
\hline All PT & 76 & 73 & 3 \\
\hline All $P M$ and $P T$ & 126 & 120 & 6 \\
\hline
\end{tabular}

sides of the chest as well as on the abdomen. PTs and PMs are not round in shape, and neither is their transillumination pattern; hence the large corona due to edema should not be mistaken for a PT or PM. In this respect, the interpretation of transillumination of the skull differs somewhat from that of the chest, in that the shape of the thoracic light pattern is of more value than is the diameter. Extensive diffraction from edema also reduces the amount of light passing through the chest wall. When this effect is combined with the larger corona previously mentioned, an existing pneumothorax or pneumomediastinum becomes obscured.

The neonate whose pneumothorax represents our third miss was a $1.8 \mathrm{~kg}$ newborn infant large for gestational age. Increased subcutaneous fat, characteristic of an infant of a diabetic mother, was noted on chest radiographs, but a glucose tolerance test had not been performed during gestation. A large $30-\mathrm{ml}$ pneumothorax in this neonate was missed by house officers using transillumination. Subsequently, a $20-\mathrm{ml}$ right pneumothorax and $30-\mathrm{mll}$ left pneumothorax were detected by us in the same patient within 12 hours, although the transmission of light was not as intense through the abnormal air collections as it has been in neonates of the same weight with normal fat thickness. One of our house officers with deutan color vision (red-green blindness) had difficulty in seeing the pneumothoraces in this neonate, even though the second and third pneumothoraces were detected by all other nursery personnel, Results of transillumination of this neonate's chest may have been affected by the experience of the examiners involved.

\section{Comments}

Chest transillumination as a tool in physical diagnosis calls for a certain degree of skill and experience Comparisons with radiographs taken at the same time are important in the development of this skill. Should there be a time separation, the results of radiography and transillumination may differ, since these air collections vary from moment to moment.

The examiner should use the same highintensity light source during each transillumination, and he should avoid shining this light at his own eyes during the procedure. An effective light shield should be fitted to the probe tip. We have found that a flexible rubber furniture leg protector works well when the closed end is excised, and pushed over the probe tip until almost flush with the end* The light probe tip must be applied perpendicular to the chest wall, particularly in the axillary areas under the pectoralis muscles, since these muscles will transmit light when the tip is placed obliquely.

Both sides of the chest should be examined in all cases. Obscuration of the chest by tape, bandages and monitor leads should be minimized. Room lights should be dimmed as much as safety allows, if only for a few seconds, particularly when the neonate is large or very edematous, or the examiner is relatively inexperienced. Examiners with deutan color vision should not rely too heavily on transillumination, although our house officer with red-green color blindness was able to see most large pneumothoraces quite well.

\section{References}

1. Kuhns, L, R., Bednarek, F, J., Wyman, M. L. Roloff, D. W. and Borer, R. C.. Diagnosis of pneumothorax and pneumomediastinum in the neonate by transillumination. Pediatrics $56: 335$, 1975.

2. Kuhns, L. R., Berger, P.E, Roloff, D.W, Poznanski, A. K., and Holt, J. F. Fat thickness in the newborn infant of a diabetic mother Radiology 3: 665 , 1974.

3. Lillard, R. L, and Allen, R. P. The estrapleural air sign in pneumomediastinum. Radiology 85: $1093,1965$.

\footnotetext{
* Devised by A. Martin, RT
} 